\title{
The perception of distance and location for dual tactile pressures
}

\author{
BARRY G. GREEN \\ Princeton University, Princeton, New Jersey
}

\begin{abstract}
The concept that distance on the skin is frequently misperceived was first reported over a century ago by Weber. Weber and others have reported that the apparent distance between pressure stimuli fluctuates with both body site and stimulus orientation. The present study confirms these effects and shows that the misperceptions are usually compressive in nature. It further establishes that errors in perceived distance correspond to errors in perceived location, indicating that an interaction exists between the perceptual processes responsible for percepts of tactile location and distance. Perceived location depends on the relationship of a tactile stimulus both to the body frame and to nearby stimuli, and the effect of nearby stimuli is to induce a perceptual affinity between sensations of pressure. These results are discussed in relation to the more frequently examined dynamic illusions of tactile distance (tau phenomenon) and location (the cutaneous rabbit).
\end{abstract}

Current psychophysical descriptions of the perception of distance on the body are incomplete. This is so despite a rich literature on tactile spatial acuity that includes the classical measures of the two-point limen and the error of localization (e.g., Boring, 1942; Franz, 1913; Friedline, 1918; Parrish, 1897; Weinstein, 1968), as well as related measures such as gap discrimination (M. B. Jones, Vierck, \& Graham, 1973), length discrimination (Davidon \& Mather, 1966; M. B. Jones \& Vierck, 1973), and size discrimination (Vierck \& M. B. Jones, 1969). In their concentration on discriminability, experimenters have generally neglected questions about the perception of suprathreshold distances.

Yet, some of the first work in what would later become sensory psychology involved judgments of the distance between two points on the skin. One of Weber's earliest contributions to psychophysics was the discovery that the perception of distance or extent was different on different parts of the body, increasing and decreasing with the spatial acuity of the area stimulated (Ross \& Murray, 1978). Early in this century, Goudge (1918), working in Titchener's laboratory, obtained results that agreed well with Weber's and showed that the perception of separation was directly related to the size of the two-point limen. Goudge's work proved to be the last analysis of

The author's present address is Department of Psychology, Indiana University, Bloomington, Indiana 47405. This research was supported by Grant NS04755-17 from the National Institutes of Health. The author thanks Roger Colewiak, Carl Sherrick, and Frank Geldard for the many helpful comments they contributed during countless discussions in the laboratory. Thanks also are extended to Martha Teghtsoonian for her constructive criticisms and comments during the review process.
Weber's illusion, and the phenomenon received no mention in Boring's (1942) review of tactile research (despite Boring's participation as an observer in Goudge's experiments). Interest in the phenomenon may have been lost because, although Weber discussed variations in perceived distance that arose from both static and moving points, Goudge (1918) reported that "with discontinuous movement the illusion is absent" (p. 118). This suggested that Weber's discovery could not be generalized to simple perceptions of distance.

However, other experiments performed without reference to Weber's illusion seem to refute Goudge's conclusion. Fitt (1917) compared the perception of separation by sight and by touch and found that the disparity between visual and tactile apparent distances depended on the part of the body that was stimulated. His proposed theory corroborated Weber's point that perceived distance covaries with spatial acuity. In the same year, Waterman (1917) demonstrated that the diameter of brass rings felt larger to the tongue than to the hand. Fifty years later, Anstis and Loizos (1967) performed an experiment similar to Waterman's, which showed that the tongue perceives small holes $(<.5$ in.) to be larger than the fingers perceive them to be.

We can conclude, therefore, that apparent distance, as defined by two tactile stimuli, varies with location on the body and seems to covary with spatial acuity. We know little else, neither the functional relationship between physical distance and perceived distance nor how this relationship may change over the surface of the body. We also remain ignorant of how the perception of distance relates to the perception of location. Given that distance is defined as the separation between two points, are the locations of 
dual tactile stimuli misperceived when apparent distance is distorted? The present study investigates these questions, both by the method of direct magnitude estimation and by methods of matching and localization. It is shown that the psychophysical functions for distance are well described by linear functions whose slopes and intercepts vary with body site and orientation of stimulation. The variations in the perception of distance found in the first experiment are discovered in the second experiment to be mostly compressive in nature. A third experiment further confirms the compressive nature of the distortions by showing that the perceived locations of two simultaneous pressure stimuli are biased toward one another.

\section{EXPERIMENT 1}

In this experiment, the method of magnitude estimation was used to construct a psychophysical scale for tactile distance.

\section{Method}

Subjects. Fifteen undergraduate students, seven women and eight men, were paid to participate; none had had prior experience in the method of magnitude estimation.

Apparatus and Procedure. Subjects were asked to assign numbers to represent the apparent distance between two simultaneous tactile stimuli. A simple hand-held stimulator was developed that provided control over stimulus location, separation, and force and enabled a high degree of flexibility of placement. The stimuli were generated with two brass rods (contactor rods) capped with cylin drical plastic tips that were $4 \mathrm{~mm}$ in diameter. The rods were inserted into 4-mm holes, drilled at $1-\mathrm{cm}$ intervals in a brass spacer bar $(2 \times 20 \mathrm{~cm})$. Only $5.5 \mathrm{~cm}$ of the length of the contactor rods passed through the holes in the spacer bar before a collar on the rods prevented them from sliding farther. The rods were inserted in the spacer bar at the desired spacing, and the bar was lowered until the contactor tips touched the skin simultaneously. Lowering the spacer bar another centimeter so caused the rods to "stand free" within the holes in the bar, thereby exerting their full $50-\mathrm{g}$ weight against the skin. Stimulation lasted approximately $2 \mathrm{sec}$.

The subject, clothed in shirt and shorts, lay supine on a mattress with eyes closed and four areas of the body exposed-palm, volar forearm, stomach, and ventral thigh. Each area was stimulated in both longitudinal and transverse orientations (longitudinal coincides with the long axis of the body). Testing took place on the right side of the body except on the stomach, where transverse stimuli straddled the midline. The four areas received stimulation in random sequence, and the precise location of stimulation on each area varied slightly from trial to trial. At the termination of stimulation, the subject responded with a number that reflected the apparent distance between the two stimuli. Subjects were urged to avoid units of inches or centimeters and received practice in assigning numbers to visual distances (the experimenter held his hands at various distances apart) before the experiment began. Because some of the test distances fell below the two-point limen, subjects were instructed that, if two distinct points were indiscernible, estimates should be based on the apparent extent of the unitary sensation.

Stimulus distances ranged from 1 to $12 \mathrm{~cm}$, with six distances tested on each area. The particular range of test distances varied across body areas according to the size of the region. Stimuli were presented randomly across regions and separations, and subjects judged each one twice in a single 1-h session.

\section{Results}

Figure 1 displays the geometric means for transverse (A) and longitudinal (B) stimulation, with body part as the parameter. The data are graphed on linear coordinates and are fitted by linear functions (correlation coefficients ranged between .989 and .998$).{ }^{1}$ A three-way analysis of variance of magnitude estimates established that the main effects of distance $[F(2,28)=243.27, p<.001]$, location $[F(3,42)=$ $29.76, p<.001]$, and orientation $[F(1,14)=16.18$, $\mathrm{p}<.001]$ were all significant, as were the interactions between stimulus orientation and stimulus location $[F(3,42)=48.57, p<.001]$, between stimulus location and distance $[\mathrm{F}(6,84)=3.31, \mathrm{p}<.006]$, and among orientation, location, and distance $[\mathrm{F}(6,84)$ $=5.46, \mathrm{p}<.001]$.

Looking first at the data for the transverse orientation (Figure 1A), it can be seen that stimuli presented to the palm and forearm produced similar perceived distances, which exceeded the distances experienced on the thigh and stomach. Individual $t$ tests show that at a separation of $2 \mathrm{~cm}$ (and above) the palm produces significantly larger estimates than either the stomach $(t=8.04, p<.05)$ or the thigh $(t=5.18$, $p<.005)$, as does the forearm at a separation of $4 \mathrm{~cm}(\mathrm{t}=2.92, \mathrm{p}<.01 ; \mathrm{t}=2.74, \mathrm{p}<.05)$. Although the slopes of the respective distance functions are positively correlated with the magnitude of perceived

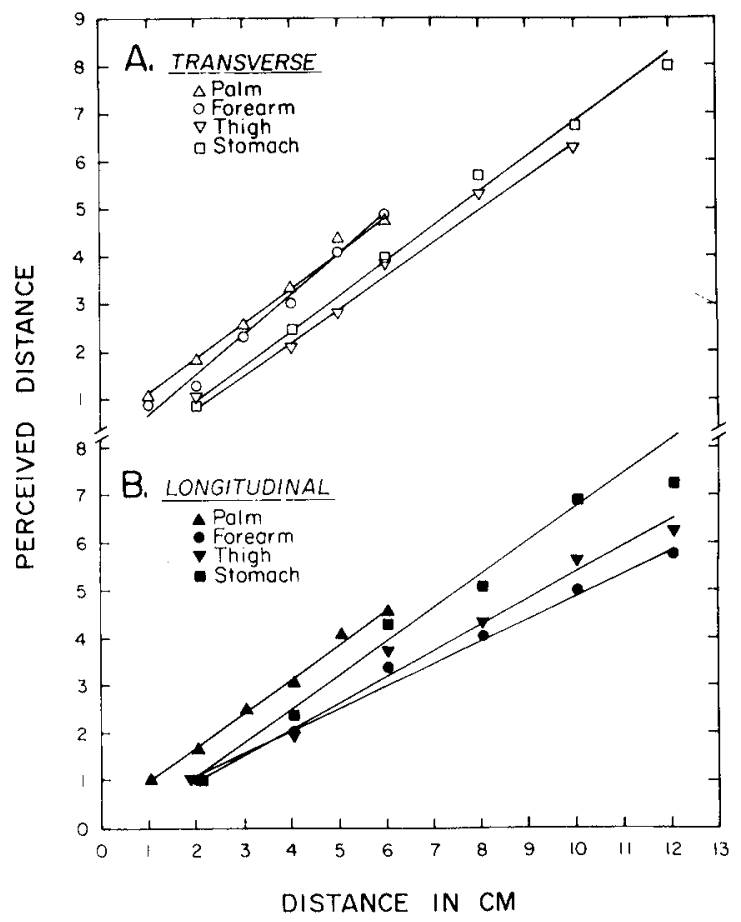

Figure 1. Geometric means of magnitude estimates of perceived distance as a function of physical distance for four areas of the body. (A) Stimulation in the transverse orientation; (B) stimulation in the longitudinal orientation. 
separation (palm $=.77$, forearm $=.81$, thigh $=.68$, stomach $=.72$ ), none reach statistical significance.

In contrast, the slopes of the distance functions in the longitudinal orientation (Figure 1B) differ significantly in all but one comparison. The palm produces a function steeper than that of either the forearm or thigh $(.73$ vs. .48 and $.55, t=10.59$ and $6.06, p<$ $.002)$, as does the stomach (.65 vs. .48 and $.55, t=$ 8.13 and $3.69, p<.002$ and .01 ). Only the palm and stomach differ insignificantly (.73 vs. .65$)$. Once again, the palm tended to produce the largest distance estimates, exceeding by significant amounts both the thigh (at $4 \mathrm{~cm}, \mathrm{t}=8.63, \mathrm{p}<.005$ ) and the forearm (at $4 \mathrm{~cm}, \mathrm{t}=9.19, \mathrm{p}<.005$ ). Whereas in the transverse orientation the forearm produced relatively large distance estimates, in the longitudinal orientation it generated some of the smallest estimates. The stomach ranked second to the palm by yielding larger estimates than either the forearm (at $6 \mathrm{~cm}$, $\mathrm{t}=3.14, \mathrm{p}<.005$ ) or the thigh (at $8 \mathrm{~cm}, \mathrm{t}=2.23$, $\mathrm{p}<.05)$.

One can conclude from these data that perceived tactile distance depends on both the body locus and the orientation of stimulation, and that the size of the orientation effect depends upon the locus. Figures 2 and 3 compare the effects of stimulating the skin along and across the body axis on the palm and stomach (Figure 2), and on the forearm and thigh (Fig-

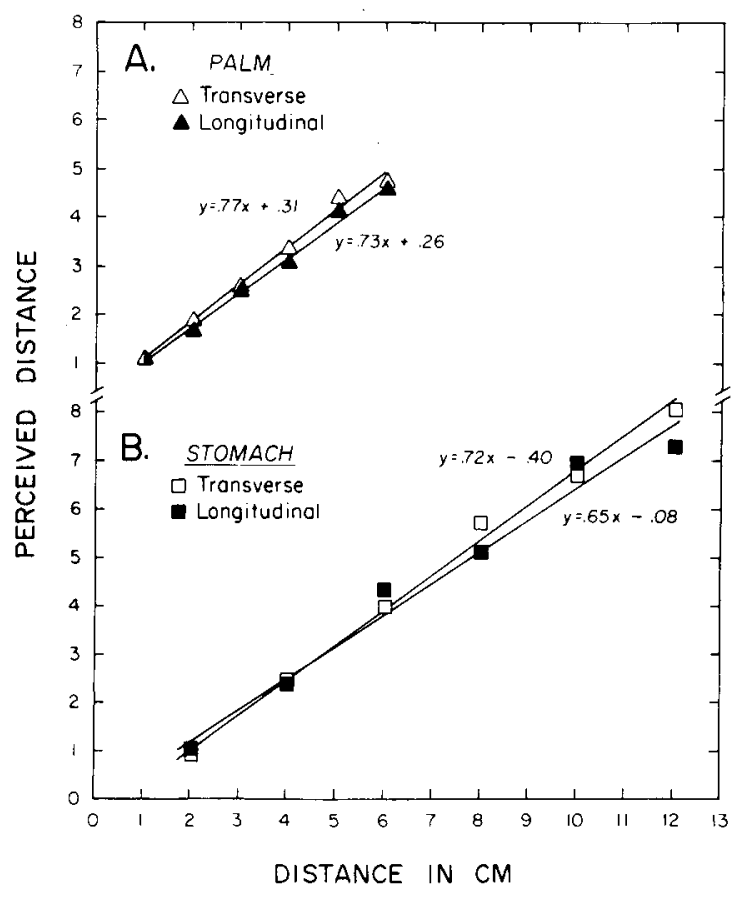

Figure 2. Perceived distance as a function of physical distance for the palm (A) and stomach (B). The parameter is the orientation of stimulation. (Equations for best-fitting linear functions are shown.)

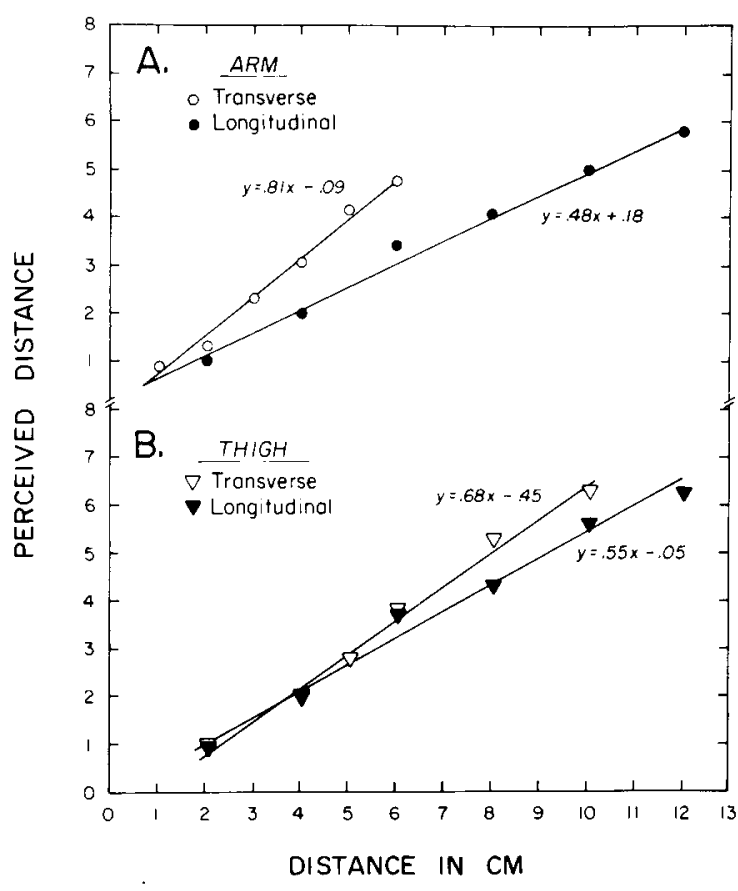

Figure 3. Same as Figure 2, for the forearm (A) and the thigh (B), showing a tendency for longitudinal judgments to be smaller than transverse judgments.

ure 3). In the former case, perceived distance remains constant across orientations; in the latter case, longitudinal distances are judged shorter than transverse distances. Hence, the two limbs appear anisotropic: the forearm shows greater anisotropy than the thigh, with distance growing $69 \%$ faster across the arm than along it. On the thigh, transverse sensations increase only $24 \%$ faster than longitudinal sensations. The regression coefficients nevertheless differ significantly between orientations on both the arm $(t=14.16$, $\mathrm{p}<.002)$ and the leg $(\mathrm{t}=5.0, \mathrm{p}<.002)$.

Weber's early conclusions having been substantiated by the discovery of differences in perceived distance on different body loci, the question arises as to which areas of the body (and which orientations) produce accurate judgments of physical distance. The results of the present experiment only hint at the answer: in both orientations, the hand, which undoubtedly has the most "experience" in assessing distances tactually, produced larger estimates of distance than most of the other areas that were tested. The hypothesis could be ventured that judgments on the palm are therefore closest to veridicality and that the lower slopes and smaller estimates encountered on other areas (particularly in the longitudinal orientation on the forearm and thigh) represent a foreshortening or compression of tactile distance. The next experiment tests this hypothesis by comparing tactile distances with visual distances. 


\section{EXPERIMENT 2}

A modulus-free magnitude estimation task like that of Experiment 1 cannot supply a direct measure of the accuracy of judged distances. The problem of obtaining unbiased estimates of distance probably cannot, however, be met simply by employing a modulus in a second magnitude estimation task. The difficulty there is that deviations from veridicality could as easily derive from misuse of the metric scale supplied by the modulus as from distortions of tactile distance. An alternative, albeit less direct, approach is to match tactile perceptions to visual distances. Vision is usually considered the dominant spatial sense (Teghtsoonian \& Teghtsoonian, 1970) and is therefore the source of most information about size, distance, and location. Furthermore, visual distance (i.e., extent in the frontal-parallel plane) has been shown, generally, to be linearly related to physical distance with a slope of unity (Stevens \& Guirao, 1963). Thus, the following assumption seems reasonable: Tactile judgments of distance can be considered accurate if the visual distances matched to them coincide with the true stimulus distance.

The forearm was selected as the test site for this experiment because of the large differences encountered there between the responses to longitudinal and transverse stimuli (Figure 3), and because of the high similarity between judgments of transverse distances on the forearm and on the palm (Figure 2A). The latter fact enables inferences to be made about the spatial properties of the palm based on data from the transverse orientation on the forearm.

\section{Method}

Subjects. Ten young adults (five men and five women) were paid to participate. None had had experience in tactile experiments.

Apparatus and Procedure. The two-point stimulator used in Experiment 1 was employed in the present experiment. The subject sat at a table, with the right arm comfortably extended, volar side up. An occluding screen positioned parallel to the arm and just to the right of the subject's head prevented the subject from viewing the forearm. The matching paradigm took the form of a production task: To the left of the screen and directly in front of the subject lay a stack of unlined paper $(20.5 \mathrm{~cm}$ long $\times 12.5 \mathrm{~cm}$ wide). The subject was told that after each stimulus presentation she/he should make two pencil marks on the paper (using the left hand) that looked as far apart as the two tactile sensations felt. The experimenter collected the response and left an unmarked sheet of paper for the next trial.

Stimuli were presented to the volar forearm in both longitudinal and transverse orientations and contacted the skin for 2 sec. Distances tested were $1,2,3,4,5$, and $6 \mathrm{~cm}$ transversely and $2,4,6$, 10,12 , and $14 \mathrm{~cm}$ longitudinally. Distances and orientations were presented in random order, and the exact site of stimulation varied slightly from trial to trial. (Longitudinal stimuli touched the skin around the midpoint of the forearm; transverse stimuli touched the skin a few inches distal to the elbow.) Subjects judged each stimulus four times in a single half-hour session, yielding a total across subjects of $\mathbf{4 0}$ estimates per stimulus.

\section{Results and Discussion}

Figure 4 displays the arithmetic means of produced visual distance for both longitudinal and transverse stimuli on linear coordinates. Consistent with the results for the forearm from the magnitude estimation task, transverse stimuli produced larger responses than did longitudinal stimuli. Evident, too, is the tendency for transverse judgments to approach veridicality (i.e., approach a slope of 1 ), whereas longitudinal judgments consistently fall short of the stimulus distances.

Some differences and similarities between the present data and those of Experiment 1 deserve mention. Comparison of the magnitude estimation data of Figure $3 \mathrm{~A}$ with the production data of Figure 4 reveals that the perceived growth of distance appears greater when subjects reproduce tactile distances visually than when they describe tactile distances numerically. Specifically, the function for perceived distance in the transverse orientation has a slope of $.81(r=.995)$ in Figure 3A, compared with $1.27(r=.981)$ in Figure 4. A similar difference between slopes (.48 and .69) was obtained in the longitudinal orientation. No obvious explanation for these discrepancies exists, although such differences are not surprising, given that the data derive from different subjective tasks. It is also notable that the estimates for the two orientations differ greatly only when a separation of $5 \mathrm{~cm}$ is reached: the first four points in the transverse orientation generally parallel the longitudinal function. The absence of a difference at small separations may be due to the size of the two-point threshold on the arm-approximately $4 \mathrm{~cm}$ (Weinstein, 1968). The variability of the estimates at and below $4 \mathrm{~cm}$ reflects

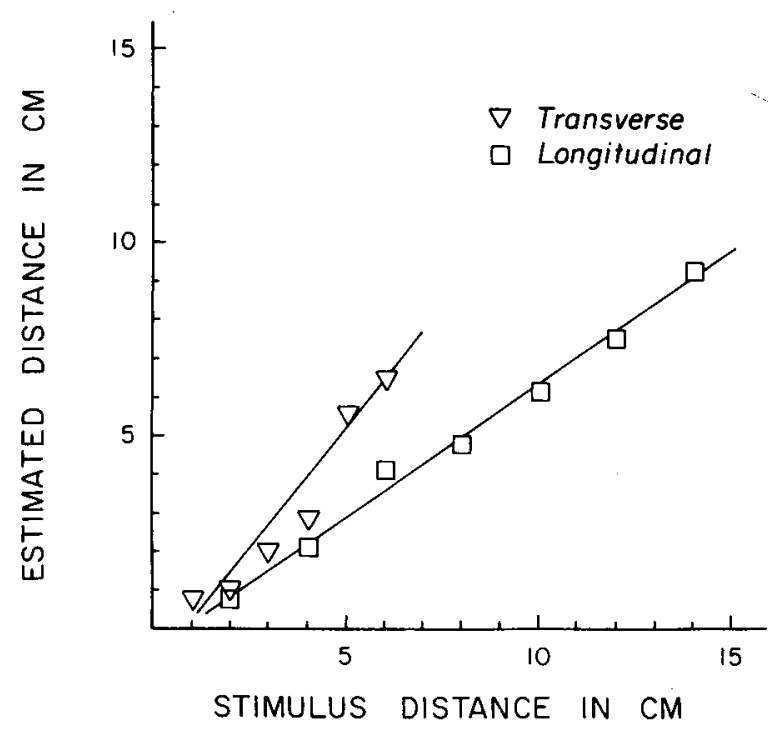

Figure 4. Reproduced distance (visual) as a function of tactile distance for transverse and longitudinal stimull on the forearm. 
the uncertainty of judgments in that region $(\mathrm{SD}=1.34$ and $1.54 \mathrm{~cm}$ at 3 and $4 \mathrm{~cm}$ ) and suggests that the apparent parallelism between functions might have occurred by chance.

Perhaps more important for the present analysis is the similarity across tasks of the relationship between slopes in the longitudinal and transverse orientations. If the transverse data of the present experiment are described by a single function of slope 1.27 , the ratio of the slopes for the transverse and longitudinal orientations stands at .54, compared with .59 in Experiment 1 . This means that whether subjects assign numbers to tactile distances or attempt to reproduce the distances visually, transverse distances are perceived as a constant proportion (.5-.6) longer than longitudinal distances. Taken together, the data from both experiments seem to indicate a compression of distance in the longitudinal orientation on the forearm. Because the transverse data lie near a slope of unity, it seems likely that very little if any "expansion" of tactile distance occurs on the forearm. It is possible, however, that other, more spatially acute areas (e.g., the tongue) may display a perceptual expansion. Experiments on the perception of the size of small holes $(<.5 \mathrm{in}$.) have provided evidence that the tongue is superior to the hand at distinguishing the size of small objects (Anstis \& Loizos, 1967). However, the present results for the forearm show that transverse distances of less than 3 or $4 \mathrm{~cm}$ are often underestimated relative to visual length, which leaves room for the tongue to exceed the "accuracy" of the forearm (and perhaps the hand) without producing an expansion of distance.

In addition to lending support to the suspicion that tactile distance is relatively compressed on some areas of the body, the present data raise fundamental questions about the relationship between perceived distance and perceived location. Given that the apparent distance between two stimuli is less than the actual distance, we might assume that the apparent locations of the two stimuli must be offset from their true sites toward locations closer to one another. This assumption rests on the logic that perceived distance and perceived location are interdependent. However, an alternative explanation is that localization remains true, but the estimation of distance falls victim to a distortion of the spatial image of the region of skin upon which the stimuli lie. For example, a distance along the forearm might be underestimated primarily because the length of the forearm is itself underestimated. In that case, a stimulus separated by the full length of the forearm might evoke a response that corresponds exactly to the perceived length of the limb, but that is several centimeters shorter than the actual length of the limb. To choose between these two explanations requires an experiment in which localization of two points is measured on a region where spatial compression seems to occur. Experiment 3 accomplishes this by asking subjects to localize stimuli along the forearm, and the results lend additional support to the concept of tactile spatial compression.

\section{EXPERIMENT 3}

This experiment provides information about tactile spatial distortions by employing a task that obviates the estimation of distance per se. Instead of estimating distance, subjects attempted to localize tactile stimuli. There were two conditions-one required subjects to localize both members of a stimulus pair, and the other required them to localize only one member of the pair. The first question is whether the localization of both stimuli reflects the compression of tactile distance that the preceding experiments revealed. If so, the processes responsible for the perceptions of location and distance must be considered nonindependent. The second question then becomes whether the mere presence of a second (adjacent) stimulus continues to affect the localization of a single stimulus. An answer would help determine the nature of any nonindependence of perceived distance and perceived location that might be found. That is, if localization is altered only when subjects attend to both stimuli (as in the dual localization condition), we can infer that the interaction depends on the perceptual process responsible for the perception of distance. If, on the other hand, localization is disturbed in both the single and dual tasks, we can infer that the interaction is more basic in nature and depends on a sensory interaction that occurs when two stimuli touch the skin in close proximity to one another.

In this paradigm, cross-modal comparisons were required to the degree that vision helped guide the subject's hand toward the perceived location of the stimuli. Visual guidance in tactile localization is, of course, typical in daily life and has long been known to produce more accurate responses than localization tasks that preclude vision (Pillsbury, 1895).

\section{Method}

Subjects. Ten university students (nine females and one male) served as paid subjects. Three (two females and one male) had served in Experiment 2, but did not know its outcome.

Apparatus and Procedure. Stimuli were generated with the hand-held device used in the preceding experiments.

The subject sat at a table, with the right arm extended as in Experiment 2. No occluding screen was present, however, because the subject was required to view the arm after stimulation. A piece of Scotch Magic Tape (approximately $1 \times 20 \mathrm{~cm}$ ) was applied along the volar forearm, just to the right of the midline. On each trial, two simultaneous pressure stimuli, $2 \mathrm{sec}$ in duration, were applied about a centimeter to the left of and parallel to the tape (i.e., on or near the midline).

In the condition that called for the subject to localize both stimuli, the location of the points varied along the arm from trial to trial, but they were never closer to the elbow or wrist than $4-5 \mathrm{~cm}$. 
This limitation avoided cues to the location of one or the other stimulus, owing to the different quality of pressure sensations that becomes noticeable in the region of the joints. In the condition that called for subjects to localize only one of the two stimuli, the location of one point in the pair was fixed throughout the testing session. The fixed stimulus touched the arm either $5 \mathrm{~cm}$ distal to the elbow or $5 \mathrm{~cm}$ proximal to the wrist, and its location was marked with chalk on the Scotch tape adjacent to it. The variable point moved randomly up and down the arm at distances of 2 to $14 \mathrm{~cm}$ from the fixed stimulus. The subjects were divided into two equal groups: for one group, the fixed point lay nearer the elbow; for the other, it lay nearer the wrist.

In the dual localization task, the procedure was the following: $A$ stimulus separation was selected and, with the subject's head turned and eyes closed, the two points were applied simultaneously to the forearm for $2 \mathrm{sec}$. Then, with eyes open, the subject made chalk marks on the tape adjacent to the apparent locations of the two stimuli. The experimenter measured the distance between chalk marks by laying a ruler next to the tape and then erased the marks by lightly rubbing the tape with a fingertip. Note that the measurements were of the distance between marks and not of the absolute error of localization. Instructions to the subjects were, however, to localize both points, and the experimenter led the subject to believe that measurements of location were being made by laying the ruler along the length of the forearm rather than simply placing the end of the ruler at one chalk mark and measuring the distance to the other. Questions directed to the subjects after the sessions revealed that they had consistently tried to localize the points in an absolute manner.

The single-point localization task, which followed the dualpoint task, proceeded similarly, except that subjects made only one mark adjacent to the apparent location of the single variable stimulus. Instructions were to focus attention on the variable stimulus and to ignore as much as possible the fixed stimulus.

Four judgments were made of each of seven stimulus separations $(2,4,6,8,10,12$, and $14 \mathrm{~cm})$ by each of the 10 subjects to yield $\mathbf{4 0}$ observations per data point in both conditions.

\section{Results and Discussion}

The data for both single and dual localization tasks on the forearm are combined in Figure 5. Each point is the arithmetic mean of $\mathbf{4 0}$ judgments. The data clearly reveal that, in the longitudinal orientation, judgments of the location of one or both points in a two-point tactile stimulus are biased toward one another, so that the apparent distance between points is less than it actually is: spatial compression is confirmed when the task is to localize stimuli rather than to judge the distance between them. The hypothesis that distortions of distance arise only from distortions in the perception of body dimensions is rendered untenable. As pointed out above, such a hypothesis predicts accurate perceptions of location together with inaccurate perceptions of distance. Thus, perceptions of distance and localization are interdependent.

Two additional facts reveal themselves in Figure 5: (1) the "error of localization" for two stimuli generally exceeds the error of localization for a single stimulus, and (2) the localization task produces two-limb functions in both conditions that were not encountered in the previous experiments in which subjects judged apparent distance.

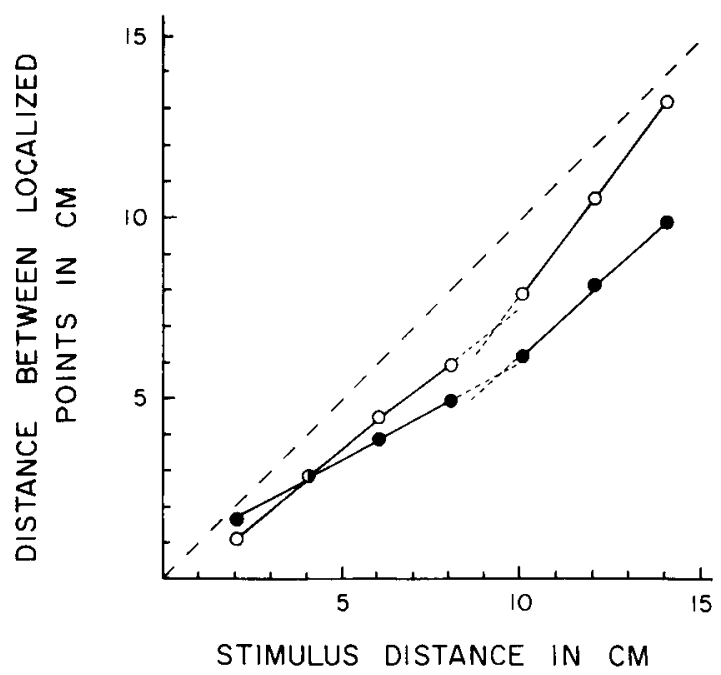

Figure 5. Distance between locallzations of tactile stimuli as a function of actual separation. Empty circles denote data from the single-point localization task; filled circles denote data from the two-point localization task.

It is notable that a difference between one- and two-point localization becomes visible only for distances greater than $4 \mathrm{~cm}$, which is approximately the size of the two-point limen on the forearm (Weinstein, 1968). Although an analysis of variance performed over the entire range of distances falls short of showing a significant difference between the single- and dual-point tasks $[F(1,9)=3.13, p<.11]$, there is nevertheless a significant interaction between the variables of distance and condition $[F(6,54)=4.64$, $\mathrm{p}<.001]$. This interaction verifies that the functions have two limbs, and an analysis restricted to the upper limbs reveals that, beyond a physical separation of $8 \mathrm{~cm}$, perceived separations are significantly larger for the single-point condition $[F(1,9)=5.23, p<.05]$.

Figure 6 shows more clearly the divergence in the results for the two conditions as physical separation grows. The error in judged separation increases in the dual localization task until a separation of $10 \mathrm{~cm}$ is reached, after which the error levels off at about $4 \mathrm{~cm}$. In contrast is the inverted U-shape of the singlepoint task, in which errors increase comparatively slowly up to a separation of $8 \mathrm{~cm}$, then decline steadily to a low of less than $1 \mathrm{~cm}$ at a separation of $14 \mathrm{~cm}$. An error of $1 \mathrm{~cm}$ falls near the expected range for the error of localization on the forearm (Weinstein, 1968).

The most significant aspect of these data is the demonstration that the perception of the location of a pressure stimulus can be biased by the mere presence of an adjacent stimulus. Because perceptions of the distance between pressures necessarily involve multiple stimuli, perceived distance may frequently derive from an interactive process that is character- 


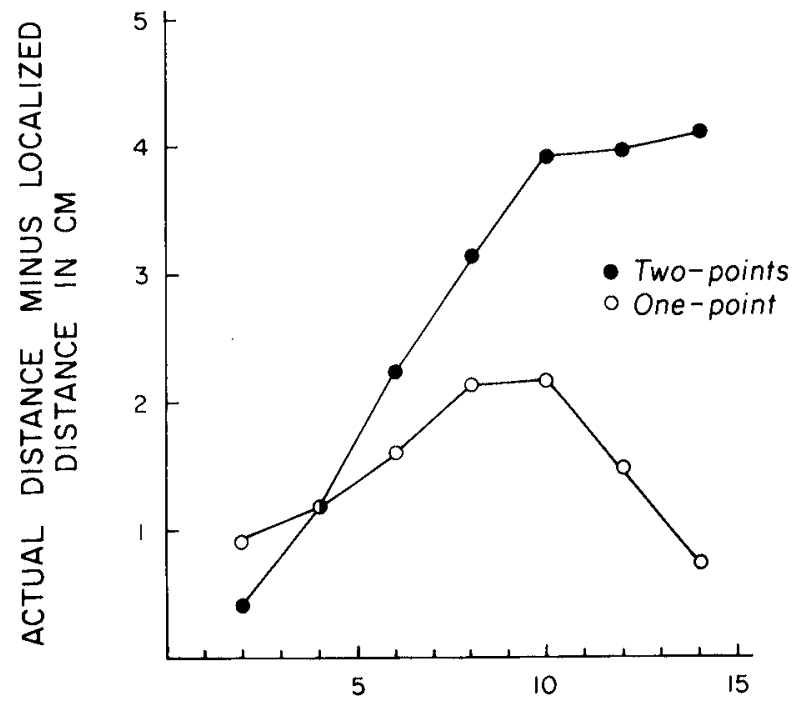

STIMULUS DISTANCE IN CM

Figure 6. The data of Figure 5 replotted to show the difference between actual stimulus separation and the apparent separation derived from the single and dual localization tasks.

ized by a perceptual affinity between sensations. That mislocations continue to occur even when the subject may (and should) ignore one stimulus of the pair indicates that the affinity results in part from a fundamental sensory and perceptual interaction between stimuli and not solely from the judgmental process required for the perception of distance. It is notable, however, that the interference produced by one stimulus upon another (single-point task) is less than that produced by two stimuli upon each other (two-point task), and that the spatial limits for interference are less in the former case $(<14 \mathrm{~cm})$ than in the latter $(>14 \mathrm{~cm})$. The necessity for the simultaneous apprehension of both stimuli appears to heighten the perceptual affinity between stimuli. Perhaps this means that the process responsible for spatial compression can be modulated by attentional variables.

Consistent with the difference in amount of spatial compression in the two conditions of the present experiment is the argument made by Boring (1942) that two qualitatively different processes give rise to judgments of absolute localization and two-point discrimination. Boring pointed out that single-point localization "measures localization with respect to the body as a frame of reference, whereas the first method (two-point discrimination) measures only the localization of one point with respect to the other" (p. 483). The same distinction may hold for the perception of distance (or two points) vs. the localization of a single point. In the two-point task, localization depends in large part upon the perceived spatial relationship between the two pressures. With this in mind, the results of the present experiment may be interpreted in the following manner: At and below the two-point limen, localization and distance perception are the same owing to the perceptual inseparability of the two stimuli. Once the limen is surpassed, localization of one stimulus in the pair begins to improve over localization of both stimuli. However, single-point localization continues to be biased centripetally. At this stage, localization seems to depend both on the relation of the stimuli to each other and to the body. As distance continues to increase, singlepoint localization improves relative to two-point localization, and, at a separation of $8-10 \mathrm{~cm}$, that improvement increases sharply. At these distances, localization may rely more heavily on the body as a referent. The improvement may be owing in part to an effect whose discovery Boring (1942) attributes to Henri, and which Parrish (1897) investigated. In that effect, a single stimulus on the forearm tends to be localized toward either the elbow or the wrist, depending on its position relative to each. We may conclude that the extent to which localization is altered by a nearby stimulus depends in part upon the extent to which judgments of location derive from the perceived relationship between the two stimuli rather than the perceived relationship between the stimulus and the body frame.

\section{GENERAL DISCUSSION}

The present study verifies the existence of tactile spatial distortions that were first reported by Weber. Modern tactile psychophysics seems to have forgotten Weber's early discoveries, and the implicit assumption has been that, beyond the two-point limen, "static" distances and locations are known with accuracy. Only transient stimuli (closely spaced in time) have been considered capable of producing distorted spatial relationships on the skin (e.g., Geldard, 1975; Helson \& King, 1931; F. N. Jones, 1956). However, nonveridical perceptions of space may be commonplace on the skin, and the existence of illusions of space by no means depends only upon dynamic spatial interactions. Because the magnitude estimates of perceived distance (Experiment 1) were similar for the stomach, thigh, and longitudinal forearm, it follows that static distance may be compressed perceptually on all of those areas. ${ }^{2}$ Whatever the mechanism is that causes spatial compression, it resides in several regions of the tactile sensory system.

It should not be surprising that static distances are frequently misperceived on the skin. Regional distortions of size (extent) occur visually as well (anisotropis), with the peripheral retina yielding smaller estimates of size and distance than the fovea (e.g., Newsome, 1972). Because perceptual distortions of 
size and distance occur in vision as well as in touch, one might conclude that construction of veridical perceptions requires specialized neural networks that serve only restricted regions of the sense organs. Such specialization could provide a structural and functional efficiency: stimuli that intrude on other areas of the sense organ need only be coarsely perceived until, if closer analysis is desired, the more acute sensory areas can be brought to bear on them.

Tactile distortions might be explained in part by Békésy's (1958, 1967) notion of sensory funneling. Funneling is believed to result from interactions between the neural processes of summation and inhibition that take place when overlapping receptive fields are activated. Békésy attempted to show how funneling might be the source of several basic sensory phenomena, acting in some cases to sharpen spatial perceptions (e.g., Mach bands), and in other cases to blur them (e.g., the two-point limen). Generally assumed to occur whenever stimulation extends over multiple receptive fields, funneling probably plays a significant role in the structuring of tactile space.

The utility of funneling as a possible explanation for tactile spatial compression might be threatened, however, by the demonstrated ability to modulate the magnitude of spatial compression by varying the demands of the task. Because spatial distortions are less in a task in which one rather than two stimuli are localized, the cause of the effect would appear to lie centrally to the level in the sensory system at which receptive fields are delimited. This assumes, however, that receptive field size (or the size of a "sensory unit") is immutable, an assumption that cannot be held with confidence. It has been demonstrated, for example, that the size of cortical somatosensory receptive fields in cats can be modulated by electrical stimulation of the pyramidal tract (Adkins, Morse, \& Towe, 1966). The presence of efferent control of tactile responsiveness makes it attractive to hypothesize that attentional variables, such as those encountered when subjects attend to one rather than both pressures, might alter the spatial extent over which neighboring stimuli interact perceptually.

Further consideration of the present data leads to the tentative conclusion that the perceptual processes that dictate spatial distortions reside more centrally in the nervous system than do the processes that delineate the character of individual sensations of pressure. This seems likely because spatial compression exists well beyond the distance at which stimuli become perceptible as separate pressures (the two-point limen); each pressure sensation seems complete with its own qualities of intensity and extensity. These "wholly formed" tactile percepts appear to have their spatial fates determined by higher order processes that integrate sensory information over a relatively large region of skin. Hence, at least two levels of processing may exist in tactile space perception: one is reflected by measures of minimum separability and spatial acuity in which stimuli have no perceptual independence; the other is reflected by a spatial affinity between seemingly independent sensations that are generated at separations beyond the two-point limen.

The present data may have implications for dynamic as well as static spatial judgments. We know that stimuli "too close" in space cannot be discriminated as separate events, and, that when separated further, cannot be accurately located. Likewise, analogous manipulations of time reveal similar spatial limitations and distortions. Together these facts imply that perception of space on the skin rests on a rather labile and relativistic foundation. The bestknown example of this lability is the tau phenomenon (Helson \& King, 1931), in which perceived distance shrinks as the time between successive tactile taps shortens. Given that the tau effect is typically measured along the forearm, it is possible that the tactile spatial compression observed with static stimuli plays a role in the illusions. Consider that simultaneous (static) tactile stimuli produce an underestimation of distance, whereas two stimuli applied independently and separated in time by several seconds produce accurate absolute localization. Now, as the time between presentation of the two stimuli is progressively shortened toward simultaneity, the expected result would be a progressive perceptual migration of the two points toward one another, causing an apparent shrinkage of distance. Viewed in this way, tau may be a manifestation of the temporal decay of spatial compression, perhaps reflecting the time course of the processes of inhibition and summation. Sensory saltation, known familiarly as the "cutaneous rabbit" (Geldard, 1975; Geldard \& Sherrick, 1972), may also be related to the static process. In saltation, tactile pulses are perceived to move toward adjacent loci that are stimulated sequentially. In the simplest situation, one tap is followed by a single additional tap (the "utterly reduced rabbit," Geldard, 1975), which results in a perceptual migration of the first tap toward the second and a shrinkage of apparent distance between taps in the manner of the tau effect. The chief difference between the cutaneous rabbit and tau may be that the rabbit results from judgments of the location of taps, whereas tau results from judgments of the distance between taps; both may be related sensorially by a common mechanism. As Experiments 2 and 3 of the present study show, subjects either underestimate distance or mislocalize stimuli, depending only on the demands of the task. A test for a common basis for these transient and static illusions requires that we assess tau, the rabbit, and static spatial compression on a variety of loci and observe whether or not the magnitudes of the il- 
lusions covary. An analysis of this kind, in which the relationships between static and dynamic perceptions of tactile space are studied, could prove fruitful in revealing the contribution of spatial and temporal factors in the structuring of cutaneous space.

\section{REFERENCES}

Apkins, R. J., Morse, R. W., \& Towe, A. L. Control of somatosensory input by cerebral cortex. Science, 1966, 153, 1020-1022.

Anstis, S. M., \& Loizos, C. M. Cross-modal judgments of small holes. American Journal of Psychology, 1967, 80, 51-58.

BÉ KésY, G., voN. Funneling in the nervous system and its role in loudness and sensation intensity on the skin. Journal of the Acoustical Society of America, 1958, 30, 399-412.

BÉ Késy, G., von. Sensory inhibition. Princeton, N.J: Princeton University Press, 1967.

Bor1NG, E. G. Sensation and perception in the history of experimental psychology. New York: Appleton-Century-Crofts, 1942.

Davidon, R. S., \& Mather, J. H. Cross-modal judgments of length. American Journal of Psychology, 1966, 79, 409-418.

Firr, A. B. The estimation of distances by sight and passive touch: Some investigations into the evolution of the sense of touch. American Journal of Psychology, 1917, 28, 264-288.

Franz, S. I. Accuracy of localization of touch stimuli on different body segments. Psychological Review, 1913, 20, 107-128.

Friedline, C. L. Discrimination of cutaneous patterns below the two-point limen. American Journal of Psychology, 1918, 29, 400-419.

Geldard, F. A. Sensory saltation. New York: Academic Press, 1975.

Geldard, F. A., \& Shenrick, C. E. The cutaneous "rabbit": A perceptual illusion. Science, 1972, 178, 178-179.

GoudGe, M. E. A qualitative and quantitative study of Weber's illusion. American Journal of Psychology, 1918, 29, 81-119.

Helson, H., \& King, S. M. The tau effect: An example of psychological relativity. Journal of Experimental Psychology, 1931, 14, 202-217.

JoNEs, F. N. Space-time relationships in somesthetic localization. Science, 1956, 124, 484.

Jones, M. B., \& VIERCK, C. J., JR. Length discrimination on the skin. American Journal of Psychology, 1973, 86, 49-60.

Jones, M. B., Vierck, C. J., Jr., \& Graham, R. B. Line-gap

- discrimination on the skin. Perceptual and Motor Skills, 1973, 36, 563-570.

Newsome, L. R. Visual angle and apparent size of objects in peripheral vision. Perception \& Psychophysics, 1972, 12, 300-304.

PARRISH, C. S. Localization of cutaneous impressions by movements without pressure upon the skin. American Journal of Psychology, 1897, 8, 250-267.
Pillsbury, W. B. Some questions of cutaneous sensibility. American Journal of Psychology, 1895, 7, 42-57.

Ross, H. E., \& Murray, D. J. E. H. Weber: The sense of touch. New York: Academic Press, 1978.

Stevens, J. C., \& Guirao, M. Individual loudness functions. Journal of the Acoustical Society of America, 1963, 36, 22102213.

Teghtsoonian, R, \& Teghtsoonian, M. Two varieties of perceived length. Perception \& Psychophysics, 1970, 8, 389-392.

VIERCK, C. J., \& Jones, M. B. Size discrimination on the skin. Science, 1969, 163, 488-489.

W ATE RMAN, C. N., JR. Hand-tongue space perception. Journal of Experimental Psychology, 1917, 2, 289-294.

WEINSTEIN, S. Intensive and extensive aspects of tactile sensitivity as a function of body part, sex and laterality. In R. Kenshalo (Ed.), The skin senses. Springfield, Ill: Thomas, 1968.

\section{NOTES}

1. Magnitude estimation data are typically fit by power functions and displayed on log-log coordinates. In the case of the present data, power functions do indeed provide good fits to the data (correlation coefficients ranged between .988 and .998 ). However, linear functions were chosen to represent the data because some of the best-fitting power functions had slopes exceeding unity, which implies positively accelerating functions rather than the linear functions observable from the data points in Figures 1 through 3. The close mathematical agreement between the two types of functions probably stems from the relatively small range of numbers on both the stimulus and response dimensions. The intercepts of the calculated functions were allowed to vary rather than being required to pass through the origin. This was done because perceived distance below the two-point limen was indeterminate. Subjects perceive a nonzero stimulus "extent" when no physical separation exists, or, conversely, they may report no separation when a physical separation persists. Because no data were collected down to and including zero physical separation, the functions were calculated from the obtained data, and lines were not drawn to the intercepts.

2. Pilot data were collected from nine subjects to learn if spatial compression occurred on the ventral surface of the thigh (longitudinally) of a magnitude similar to that found on the forearm. The procedure was the same as the two-point task of Experiment 3. The results confirmed the existence of compression on the leg, with distance being underestimated by $1.7 \mathrm{~cm}$ for a 4-cm separation and by $4.4 \mathrm{~cm}$ for a $14-\mathrm{cm}$ separation. These numbers agree well with the errors for the same separations on the forearm.

(Manuscript received April 29, 1981; revision accepted for publication January $21,1982$. 